\title{
Tratamiento multivariado de subpoblaciones en una gran encuesta social: la construcción de zonas sociales
}

\author{
Carlos Lozares ${ }^{1}$ \\ Màrius Domínguez i Amorós 2
}

1. Universitac Autonoma de Barcelona. Deparcament de Sociologia. 08193 Bellaterra (Barcelona). Spain

2. Universitat de Barcelona. Departament de Sociologia i Metodologia de les Ciències Socials. 08034 Barcelona. Spain

\section{Resumen}

Cuando se realiza una encuesta social en un amplio territorio queda siempre el deseo de aplicar análisis similares a los realizados en la encuesta a poblaciones o territorios más reducidos, evidentemente utilizando los propios datos de la encuesta.

El objetivo de este artículo consiste en mostrar cómo cada estrato de una muestra estratificada puede consticuir una base muestral para llevar a cabo dichos análisis con todas las garantías de precisión o, al menos, con garantias calculables y aceprables sin aumentar el número muestral para la encuesta general.

Palabras clave: metodología, muestreo estratificado, análisis de correspondencias, análisis de clasificación, zonas sociales.

Abstract. Multivariate processing of subpopulations by social survey: the construction of social zones

When one carries out a Social Survey in a wide population, one would like to carry out the same analysis with a small population, using, of course, the same data of the Survey.

The aim of this paper is to show each stratum of a stratified sample can be a sample population which allow an analysis with the reliable, or at least, calculable and acceptable guarantees without increasing the sample number of the general Survey.

Key words: methodology, stratified sample, analyse of correspondence, social zones.

\section{Sumario}

\section{Introducción 7. Comentario finzl}

2. El proceso general seguido

3. Los errores de cada estrato muestral y su transformación en zonas sociales.

El perfil de las zonas sociales

4. La estructura dimensional de cada zona

5. Los grupos sociales en cada zona social

6. Algunas conclusiones generales referentes a la estructura dimensional $y$ a los grupos sociales
Anexo 1. Distribución de las zonas sociales en la región

Anexo 2. Elección de variables

Anexo 3. Perfil de las zonas sociales

Anexo 4. Ilustración de la distribución de las zonas sociales en el distrito 6: Gràcia

Referencias 


\section{Introducción}

El estudio presentado en este artículo se enmarca en los análisis realizados en la Enquesta de la Regió Metropolitana de Barcelona 1990. Nos remitimos pues a los volúmenes publicados de la misma y, más concretamente, a los que aparecen en la bibliografía final $y$, en particular, en la referencia que se hace a dicha encuesta en uno de los artículos de este mismo número de Papers.

Cuando se realiza una encuesta social en un amplio territorio queda siempre el deseo, a veces satisfecho, de aplicar análisis similares a los realizados en la encuesta, u otros diferentes, a poblaciones o territorios más sectoriales. El problema que surge es que suelen ser irrealizables dado que el error de tales poblaciones o territorios no es admisible por ser excesivamente grande dentro de lo habitualmente aceptable. Evidentemente esto se hubiera podido solucionar previamente si se hubieran elegido errores convenientes para dichos territorios o poblaciones calculando en función de ellos el error de la muestra general pero con el consiguiente elevado número de muestra que haría inviable la realización de la encuesta.

Nos proponemos mostrar cómo el muestreo estratificado, utilizado para construir la muestra, permite llevar a cabo análisis en territorios y poblaciones más reducidos que los de la encuesta general con todas las garantías de precisión o, al menos, con garantías calculables y aceptables sin aumentar el número de muestra previsto para la encuesta general. El procedimiento consiste en aprovechar los estratos territoriales de dicha muestra estratificada para que sean soportes poblacionales más reducidos sin que los errores sobrepasen límites inaceptables. Es obvio que a menor error en la encuesta general corresponderá un menor error en cualquiera de sus territorios y poblaciones, pero, en igualdad de condiciones, la muestra estratificada consigue ganancias adicionales para realizar la operación que proponemos.

El principio en que se basa este procedimiento es sencillo. Los estratos de una muestra estratificada son partes del territorio (o de la población) en el que (o sobre la que) se realiza la encuesta general. En el objetivo de la construcción de la muestra estratificada está la idea básica de que cada estrato ha de ser lo más homogéneo posible con relación a las variables criterio que sirven para calcular la muestra. En consecuencia, se construyen los estratos de tal manera que la dispersión y, por tanto, la desviación de cada uno sea la menor posible. Por tanto, el territorio o población que abarca cada estrato tendrá también el menor error posible, pues depende de la desviación. Evidentemente, no entraremos aquí en el detalle de la construcción de la muestra y de los estratos, y mucho menos en su descripción (Carlos Lozares, Pedro López, 199 la.; Carlos Lozares, Pedro López, 1991b).

Pretendemos, pues, dar cuenta det proceso metodológico seguido para conseguir los objetivos que acabamos de describir. Las reflexiones que hacemos sobre los aspectos de contenido tienden a poner de manifiesto los resultados y la eficacia del método.

Veamos previamente algunos conceptos o términos utilizados. Hemos men- 
cionado anteriormente los estraros muestrales. Como ha quedado claro, son conjuntos territoriales o poblacionales más reducidos que el territorio o población en el que se realiza la encuesta general. Se calculan y se hallan en el proceso mismo de la construcción de la muestra estratificada. Por el mismo proceso de su construcción cada uno de estos conjuntos es lo más homogéneo posible. Los estratos muestrales son conjuntos de secciones censales que son las unidades utilizadas para construir la muestra estratificada. Ello hace que los estratos puedan ser geográficamente localizables. Lo que denominamos zonas sociales son los mismos estratos, pero a los que se les aplica los datos correspondientes de la encuesta general. Evidentemente, el conjunto de las zonas sociales llenan así toda la Región I Metropolitana, que es el territorio en el que se ha realizado la encuesta. Cada zona será objeto de los análisis sociológicos convenidos si tienen, como veremos, niveles de error aceptables.

\section{El proceso general seguido}

He aquí un esquema del proceso.

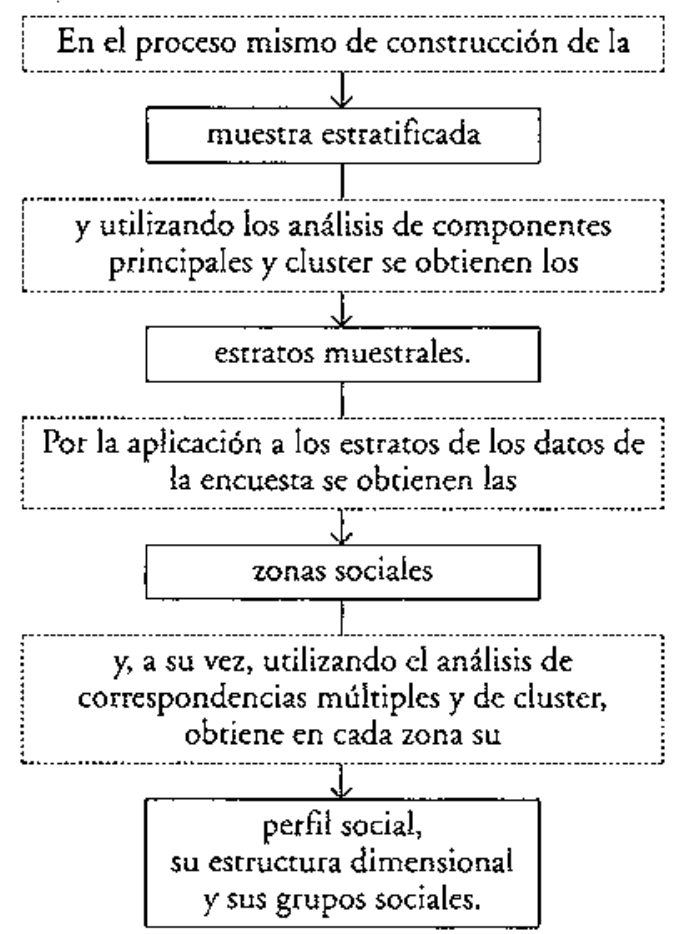

Como acabamos de ver en el esquema precedente, para construir las zonas sociales se parte de los estratos de la muestra estratificada. Daremos breve cuen- 
ta de los errores correspondientes de cada estrato que serán los de las zonas sociales a fin de asegurar su representatividad muestral a la hora de aplicar los datos de la encuesta a cada uno.

Por la aplicación de las encuestas realizadas en cada estrato muestral, éstos se convierten en lo que hemos llamado zonas sociales. En ellas se pueden hacer los análisis que se crean convenientes. Los realizados por nosotros corresponden a los perfiles sociales, la estructura dimensional y los grupos sociales de las zonas sociales.

Por la estructura dimensional tratamos de conocer las dimensiones que más y mejor explican la diversidad de la población de cada zona en las variables elegidas, esto es, aquellos ejes que más discriminan a su población. Por los grupos sociales damos cuenta de cómo se agrupa la población de la manera más homogénea posible con relación a la estructura dimensional en cada zona. Estos dos últimos análisis son paralelos a los realizados en la encuesta general para toda la población (Marina Subirats, Cristina Sánchez, Màrius Domínguez, 1992).

\section{Los errores de cada estrato muestral y su transformación en zonas sociales. El perfil de las zonas sociales}

La primera parte del proceso de análisis ha consistido, pues, en retomar los estratos muestrales de la muestra estratificada. En su momento, se habían identificado ocho estratos. En su transformación en zonas sociales se consideran solamente siete debido a la agregación de dos de ellos por las razones que veremos a continuación. La primera cuestión consiste en saber si cada uno de los estratos es suficientemente consistente desde el punto de vista muestral.

En la tabla I aparecen una serie de datos sobre los estratos muestrales, número de secciones que le corresponden, número de encuestas realizadas en él, población de más de dieciocho años, distancia media de cada sección al centro de inercia de cada estrato, varianza, desviación típica y error correspondiente para el número de encuestas realizadas.

Como vemos en la mayor parte de los estratos, los errores son aceptables para tratar cada uno como una unidad poblacional. Las encuestas realizadas en cada uno son representativas de su población en la medida que lo indica su error correspondiente. Con todo, el error de 7,5\% para el estrato 2 es inaceptable; además, habrá que ser prudente con las afirmaciones que se hagan en los estratos 4 y 7 , ya que sus errores son, respectivamente, del $4,4 \%$ y del $5,6 \%$. Para resolver el problema del estrato 2 se ha unificado con el estrato 1. Rehaciendo los cálculos para estos estratos agregados el error se rebaja al 2,8\%. Ello nos permite tratar lo que llamariamos un nuevo estrato $1+2$ con todas las garantías. Esta agrupación está plenamente justificada desde el punto de vista de los contenidos, ya que, analizados independientemente, ambos estratos a partir de los datos de la encuesta, poseen características similares. Ver de nuevo la tabla 1 para los datos de ambos agrupados.

En el anexo 1 aparece el número de secciones censales por municipios y por zonas sociales en la Region I Metropolitana de Barcelona. Evidentemente, 
Tabla 1. Serie de datos para cada uno de los estratos muestrales.(1) Estrato. (2) Núm. de secciones censales. (3) Núm. de encuestas realizadas. (4) Población de más de dieciocho años. (5) Distancia media de cada sección al centro de inercia de cada estrato. (6) Varianza. (7) Desviación típica. (8) Error para un $95 \%$ de confianza.

\begin{tabular}{lccllllll}
\hline$(1)$ & $(2)$ & $(3)$ & $(4)$ & $(5)$ & $(6)$ & $(7)$ & $(8)$ & en $\%$ \\
\hline 1 & 173 & 671 & 230.475 & 1,871 & 0,750 & 0,870 & $0,0350(3,5 \%)$ \\
2 & 53 & 219 & 53.844 & 2,142 & 1,480 & 1,217 & $0,0750(7,5 \%)$ \\
3 & 909 & 865 & 690.586 & 0,954 & 0,109 & 0,331 & 0,0245 & $(2,4 \%)$ \\
4 & 350 & 432 & 258.580 & 1,050 & 0,238 & 0,487 & 0,0442 & $(4,4 \%)$ \\
5 & 654 & 906 & 725.118 & 0,947 & 0,138 & 0,371 & 0,0255 & $(2,5 \%)$ \\
6 & 664 & 810 & 610.572 & 0,917 & 0,148 & 0,385 & 0,0292 & $(2,9 \%)$ \\
7 & 275 & 351 & 167.188 & 1,160 & 0,398 & 0,631 & $0,0566(5,6 \%)$ \\
8 & 431 & 807 & 441.417 & 1,281 & 0,299 & 0,547 & $0,0293(2,9 \%)$ \\
& 3.509 & 5.061 & 3.177 .763 & & & & & \\
$(1)$ & $(2)$ & $(3)$ & $(4)$ & $(5)$ & $(6)$ & $(7)$ & $(8)$ & $(\%)$ \\
\hline $1+2$ & 226 & 890 & 284.302 & 1,281 & 0,299 & 0,547 & 0,0280 & $(2,8 \%)$ \\
\hline
\end{tabular}

los límites no coinciden con los administrativos de las comarcas, municipios, distritos o barrios. La agrupación de las secciones censales por estratos viene de la homogeneidad que en ellos tienen las características de las variables q̨ue sirvieron para construir la muestra estratificada, es decir, de los datos del censo.

Como decíamos precedentemente, las zonas sociales no son más que los estratos a los que hemos aplicado los datos de la encuesta. No hemos trabajado con todas las variables de la encuesta metropolitana, sino con una serie de variables elegidas. Concretamente, se han retomado las mismas que en el estudio de los grupos sociales aplicado a toda la Región I (Marina Subirats, Cristina Sánchez, Màrius Domínguez, 1992). Además, en el artículo precedente se da cuenta de dicha selección de variables. Con todo mostramos una descripción breve en el anexo 2 .

El primer estudio realizado en las zonas sociales es su perfil social. Hablaremos muy brevemente de proceso de análisis, ya que los resultados más característicos han sido objeto de un tratamiento más detaliado en uno de los monográficos de la Enquesta de la Regió Metropolitana 1990 (Carlos Lozares, Màrius Domínguez, 1993). Este proceso es descriptivo por análisis bivariado al comparar las zonas entre sí y con toda la Región. I como marco de referencia general. Por consiguiente, en este análisis no se toma cada zona social como universo poblacional y, por consiguiente, muestral. Lo más característico o modal de cada zona social, y por tanto su perfil social, es lo que más la diferencia de las otras. En el anexo 3, aparece un resumen de dichos resultados.

Las zonas sociales pueden mapificarse siguiendo las secciones censales que pertenecen a cada zona. Como ejemplo del interés del tratamiento que presentamos hemos elegido el distrito 10 de Gràcia, en el anexo 4. 


\section{La estructura dimensional de cada zona}

Realizado el análisis del perfil descriptivo de cada zona — recordemos que dicho análisis se ha hecho comparando las zonas entre sí sin considerarlas como unas poblaciones muestrales independientes - el siguiente paso ha consistido en construir lo que denominamos la estructura dimensional de cada zona.

Por estructura dimensional de la zona entendemos la organización del conjunto de los datos/variables elegidos para cada zona en ejes o variables más densos de contenido, combinación lineal de las primeras variables, que den cuenta del mayor porcentaje posible de la varianza total sin que ello suponga una excesiva pérdida de información. Para ello se ha utilizado también el análisis de correspondencias múltiples. La interpretación de dichos ejes acostumbra a ser un trabajo laborioso. Para poder entrar en el análisis de la estructura dimensional de las zonas sociales es imprescindible que el error muestral sea aceptable en cada zona, puesto que cada una se toma ahora como población muestral.

Para llegar a dicha estructura dimensional, ha sido necesario realizar un paso intermedio, es decir, dimensionalizar cada uno de lo que hemos denominado ámbitos sociales y construir una tipología en cada uno y para cada zona.

I. Dimensionalizar cada uno de los ámbitos de las variables y construir en cada uno una variable tipológica que dé cuenta de la agrupación de la población de la zona en cada ámbito.

No insistiremos demasiado en este objetivo intermedio, pues el método ha sido idéntico al explicado anteriormente para hallar las dimensiones de la estructura de la población total.

Un ámbito está definido por un conjunto de variables elegidas que corresponden a fenómenos sociales que tienen una cierta coherencia y autonomía sociales, sea por darse en un mismo cuadro institucional/organizacional, sea porque son "próximos" los espacios o tiempos sociales en que se dan, p.e., el conjunto de variables referentes al trabajo, a la educación, a la vivienda, etc. ${ }^{1}$. La necesidad de esta fase previa en cada zona obedece básicamente a una razón de simplificación y racionalización del proceso: realizar un análisis con 742 modalidades (más dos contintras) nos hubiera conducido más bien a una confusión real que a una clarificación real.

2. Hallar la estructura dimensional para el conjunto de las tipologías de los ámbitos para cada zona social.

Por la fase precedente disponemos de once variables tipológicas en cada una de las siete zonas sociales retenidas. Cada una de las once tiene unas

1. Los ámbitos retenidos son los siguientes: trabajo; trabajo producrivo y reproductivo; rique2a del hogar; equipamiento del hogar; residencia; educación; relaciones personales; tiempo libre; hábitos de consumo; composición y relaciones familiares; vacaciones; origen geográfico. (Ver de nuevo el anexo 2 para una descripción más detallada). La identidad de estos ámbitos, los resulcados de su dimensionalización y las ripologias consrruidas en ellos para cada zona no son más que los resultados de los mismos procesos aplicados a toda la Región I. 
cuatro o cinco modalidades. Se trata ahora de encontrar unas nuevas dimensiones en cada zona a partir de las once variables tipológicas, o mejor, a partir de todas sus modalidades, es decir, repetir lo que se había hecho en toda la Región I pero ahora en cada zona. Por tanto, dichas tipologías han sido tratadas como variables activas $y$, con las variables estructurales como ilustrativas, son las que se han empleado para dimensionalizar cada una de las zonas sociales. El método seguido ha sido de nuevo el análisis de correspondencias múltiples.

La estructura dimensional da cuenta de los contenidos más explicativos del conjunto de los valores de las variables que definen cada una de las zonas. Es como el esquema o esqueleto sustantivo de la misma.

3. Los resultados de la estructura dimensional de las zonas.

Daremos solamente una visión esquemática de los resultados a fin de mostrar la eficacia del método seguido.

En general con cinco dimensiones retenidas se superaba el $60 \%$ de la varianza en cada una de las zonas sociales analizadas. A la hora de identificarlas lo hemos hecho solamente de las dos primeras. La tercera presentaba ya algunas dificultades interpretativas. Con todo, en algunas zonas hemos llegado a identificar tres.

Se puede afirmar que se da una gran semejanza en los contenidos de la dimensiones de todas las zonas $y$, por tanto, que bajo la diversidad manifiesta de sus perfiles sociales aparecen unas formas de estructura dimensional que solamente difieren en la variabilidad explicada por cada dimensión cn cada zona. Este hecho es importante, pues nos permite hacer una lectura más simplificada y en código unificado del conjunto de las zonas que no es más que la traducción de la estructura dimensional de toda región en la variedad de cada zona. No entraremos en explicar con detalle la identidad de cada una de las dimensiones en cada zona, ya que no es el objetivo de este artículo. Simplemente expresaremos esquemáticamente cual es el modelo más habitual de dimensionalización extraído de los resultados de cada una de las zonas. Solamente hacemos una descripción de las dos dimensiones, que aparecen en todas las zonas.

- La primera dimensión viene dada por el conjunto de valores que se expresan a continuación. (Se entiende que los valores de un lado se oponen a los del otro dentro de la dimensión y que dicha polaridad es la que contribuye a definirla.)

Edad: 26 a 45

Más presencia del hombre

Alto estatus socioeconómico y cultural

Familia nuclear reducida

Origen catalán (o mixco)

Nivel elevado en todos los ámbitos
Edad: + de 65 (a veces +55)

Más prescncia de la mujer

Bajo (a veces muy bajo) estatus socioeconómico

Hogates unipcrsonales

Origen no caralán

Nivel bajo en todos los ámbiros 
- La segunda dimensión viene dada también por el conjunto de los valores que se expresan a continuación

\begin{tabular}{ll}
\hline Edad: más fuerza los 35 a 55 & $\begin{array}{l}\text { Edad: sobre todo de más } \\
\text { de } 65 \text { y menos de } 35\end{array}$ \\
$\begin{array}{l}\text { Algo más presencia del hombre } \\
\text { Estazus social y cultural }\end{array}$ & Algo más presencia de la mujer \\
$\quad$ medio-medio bajo & Estatus social y cultural medio-alto \\
Familia nuclear & Hogares unipersonales \\
Origen no catalán & Origen catalán \\
Nivel medio-bajo en & Nivel medio-alto en todos \\
todos los ámbitos & los ámbitos
\end{tabular}

La intensidad de cada dimensión (varianza explicada) en cada zona es diferente. Incluso hay en alguna zona una tercera dimensión que posee más varianza que ambas. pero estas dos están presentes en todas las zonas. La originalidad y especificadad de cada zona está dada por la manera de combinarse en prioridad las dos y con una tercera no que varía según la zona.

\section{Los grupos sociales en cada zona social}

Los grupos sociales de cada zona se constituyen clasificando automáticamente los individuos en función de las dimensiones retenidas en ellas. Veamos el proceso seguido.

Los grtupos sociales buscados habrán de tener suficiente significación en el interior de la zona. Hemos mantenido la idea de construir el menor número de ellos: dos, tres, o a lo sumo cuatro, para poder retener un volumen frecuencial suficiente. Para dicha construcción se han utilizado, en cada zona, los cinco ejes o dimensiones obtenidos en el análisis precedente del análisis de correspondencias. El método de análisis ha sido el de cluster. La descripción e identidad de los grupos sociales de cada zona social han sido hechas por análisis bivariado de las variables estructurales y de los ámbitos y por su proyección en los ejes explicados. Los grupos sociales de cada zona se contemplan y se analizan comparativamente teniendo la zona como referencia global. Veamos algunos de los resultados más remarcables de este análisis en vistas precisamente a mostrar el interés y la eficacia del método seguido.

1. Es relevante la tendencia a que el mágico número de tres satisfaga en el análisis de cluster las particiones en cada zona. En todas ellas los razonamientos abocan a elegir esta cantidad como óptima. Hay variedad, pero evidentemente no tan extensa como en toda la Región I. Todos los intentos de extenderse a cuatro o cinco grupos ha conducido al nacimiento de Grupos poco significativos dentro de cada zona.

2. La variación de la identidad de los grupos sociales en cada zona proviene sobre todo del peso diferenciado que tienen las dimensiones precedentes 
en cada zona que dan también un peso distinto a la variedad no excesivamente grande de los grupos sociales posibles.

3. Otro hecho importante aunque esperado consiste en que el Perfil de la zona coincide con el grupo social más modal y, en la casi totalidad de zonas, con el más cercano al centro de inercia de la zona. Si hay dos grupos con fiecuencias altas y casi similares, el perfil es una combinación de ambos. Lo que es interesante es que por debajo del Perfil social subsiste y se manifiesta una realidad social que no aparecería de no hacer este análisis independientemente en cada zona. Por ejemplo el grupo representado por los mayores de edad, los inactivos, las mujeres, los hogares unipersonales, el bajo estatus socioeconómico y cultural y el origen no catalán está presente en todas las zonas, aunque no es mayoritario en ninguna zona salvo en la siete.

\section{Algunas conclusiones generales referentes a la estructura dimensional y a los grupos sociales}

I. Aparece claramente manifiesta la función estructurante que en todas las zonas sociales y grupos juegan tas categoría socioeconómica y/o socioprofesional, en general el estatus socioeconómico y cultural (CSE, CSP, actividad, ocupación, situación en mercado del trabajo, nivel educativo y prácticas culturales, etc.). Se suele acompañar, evidentemente, de los ingresos del hogar en sus diversas manifestaciones. Con todo, no siempre estos factores son los de más peso en alguna de las zonas. A veces es el hábitat, en sus múltiples concreciones (vivienda, segunda residencia, barrio), el más evidente; otras, el equipamiento del hogar y el consumo.

2. En casi todos los grupos de las zonas las variables ligadas a la movilidad y a la radicación en el barrio (año de construcción de la vivienda, años de llegada al mismo o al municipio, dimensión del municipio, piso deseado, razón de cambio de vivienda) intervienen en los perfiles aunque no tanto en la configuración de los grupos. Por contra, el origen individual, de Cataluña o no, que está presente en la estructura dimensional, es un clemento que pierde fuerza en la configuración de algunos de los grupos con relación a otros valores de la variable origen que adquieren más peso como el "de dentro y fuera de Cataluña" de los miembros del hogar, los matrimonios mixtos y el bilinguismo.

3. Dos factores sociodemográficos, la edad y el sexo, aparecen claramente a la hora de configurar las zonas y los grupos, y ambos con fuerte incidencia.

4. Cuanto más se haya llegado a la madurez productiva, a la plena ocupación laboral, a una mayor cualificación -sea ésta de obrero cualificado o sca, sobre todo, a partir de técnico medio-, cuanto más elevado sea el estatus socioeconómico y cultural y se disponga de más tiempo libre y de vacaciones, más masculinizado aparece el perfil del grupo social, y al conera- 
rio. He aquí una constante permanente de la configuración de los grupos sociales de las zonas.

5. Este tipo de análisis puede prestarse también a estudiar la capacidad evolutiva de las zonas. Hay zonas que por sus características manifiestan una aparente propension a una permanente e imperturbable reproducción. Otras se muestran más dinámicas observando los grupos que las componen. En este sentido, hay algunas de las zonas que por la naturaleza de su perfil y de sus grupos, por su dinámica estructural, por sus índices indudables de modernidad, por la influencia de los factores culturales, por su nivel técnico-profesional, por su mezcla generacional y de origen, por su edad y movilidad social y geográfica y por su nivel relacional y asociativo, manifiestan más dinamismo social y más capacidad innovadora. Las hay que se mantendrán precariamente o en un progresivo declive dada la edad de sus individuos y la escasez de gente joven en ellas. Pero al hacer estas afirmaciones no solamente nos referimos a la edad como factor fundamental, sino también a otras características ligadas a los aspectos socioprofesionales, a la movilidad geográfica, etc.

\section{Comentario final}

Esta visión de la región a partir de las características de las zonas como una aglomeración de realidades plurales, no precisamente simples, es de gran interés. Sea en análisis genéricos, sea en análisis de estructura social de una región, se puede llegar a tener una visión excesivamente abstracta, de tiralíneas y manual social, de un territorio. La vuelta a una realidad, localizable geográficamente y más desmembrable como las zonas, nos permite ver un mapa más variado en sus calles y secciones censales y descubrir además en el interior de cada zona una nueva variedad. Cada zona se nos aparece diferente en sus perfiles pero, dentro de su homogeneidad, también hay una estructura y de la comparación entre las diferentes zonas surgen unos modelos generales de composición de grupos y la idea de una cierta dinámica social. 
Anexo 1. Distribución de las zonas sociales en la región.

El cuadro que viene a continuación representa el número de las secciones censales en cada municipio pertenecientes a cada zona. Aparecen ocho zonas y no siete, pues están desagregadas la I y la 2 .

\section{Distribución del número de secciones en cada municipio según las diferentes zonas sociales}

$$
\begin{array}{llllllll}
\text { Zonas } & & & & & & \\
\hline 1 & 2 & 3 & 4 & 5 & 6 & 7 & 8
\end{array}
$$

\section{Baix Llobregat}

\begin{tabular}{|c|c|c|c|c|c|c|c|}
\hline 001 Abrera & 2 & 0 & 0 & 0 & 0 & 0 & 0 \\
\hline 020 Begues & 0 & 1 & 0 & 0 & 0 & 0 & 0 \\
\hline 056 Castelldefels & 0 & 0 & 0 & 0 & 3 & 5 & 0 \\
\hline 066 Castellyi de Rosanes & 0 & 1 & 0 & 0 & 0 & 0 & 0 \\
\hline 068 Cervelló & 3 & 0 & 0 & 0 & 0 & 0 & 0 \\
\hline 069 Collbató & 0 & 1 & 0 & 0 & 0 & 0 & 0 \\
\hline 072 Corbera de Llobregat & 0 & 1 & 0 & 0 & 0 & 0 & 0 \\
\hline 073 Cornellà de Llobregat & & 0 & 4 & 0 & 50 & 15 & 0 \\
\hline 076 Esparteguera & 5 & 0 & 1 & 0 & 2 & 1 & 0 \\
\hline 089 Gavá & 0 & 0 & 2 & 0 & 18 & 0 & 0 \\
\hline II 4 Martorell & 4 & 0 & 1 & 0 & 3 & 1 & 0 \\
\hline 123 Molins de Rei & 0 & 0 & 5 & 0 & 3 & 2 & 0 \\
\hline 147 Olesa de Montser rat & 3 & 0 & 1 & 0 & 3 & 0 & 1 \\
\hline 157 Pallejà & 3 & 0 & 0 & 0 & 0 & 0 & 0 \\
\hline IS8 El Papiol & 2 & 0 & 0 & 0 & 0 & 0 & 0 \\
\hline 169 El Prat de Liobregat & I & 0 & 3 & 0 & 26 & 7 & 0 \\
\hline
\end{tabular}

$$
\text { Cervelló }
$$

196 Sant Andreu de :

$$
\text { a Barca }
$$

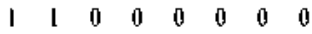

$\begin{array}{llllllll}0 & 0 & 0 & 0 & 5 & 1 & 0 & 0\end{array}$

200 Sant Boi de Llobregat $0 \begin{array}{llllllll}0 & 0 & 2 & 0 & 36 & 5 & 0 & 1\end{array}$

204 Sant Climent de

Llobregar

$\begin{array}{llllllll}0 & 1 & 0 & 0 & 0 & 0 & 0 & 0\end{array}$

208 Sant Estere Sesroyires $0 \quad 1 \quad 000001000$

211 Sant Feliu de

Llobrcgat

217 Sant Joan Despi

263 Sant Vicens

dels Horts

289 Torréles de

Ulobregat

295 Valliranz

301 Viladecans $\begin{array}{llllllll}0 & 0 & 3 & 0 & 21 & 2 & 0 & 0\end{array}$

$\begin{array}{llllllll}0 & 0 & 0 & 0 & 11 & 0 & 0 & 2\end{array}$

$\begin{array}{llllllll}1 & 0 & 0 & 0 & 8 & 4 & 0 & 0\end{array}$

$\begin{array}{llllllll}0 & 1 & 0 & 0 & 0 & 0 & 0 & 0\end{array}$

$\begin{array}{llllllll}2 & 0 & 0 & 0 & 0 & 0 & 0 & 0\end{array}$

$10000022 \quad 1 \quad 00$
21 Maresme

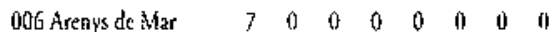

007 Árenys de Munt $4 \begin{array}{lllllllll}4 & 0 & 0 & 0 & 0 & 0 & 0 & \|\end{array}$

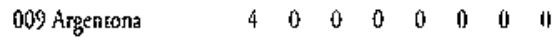

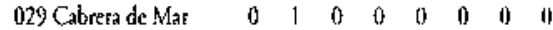

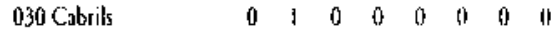

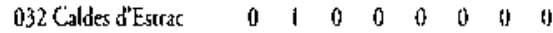

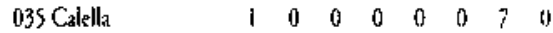

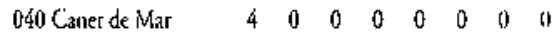

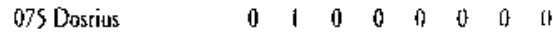

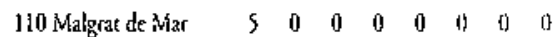

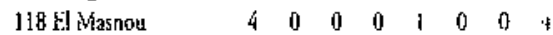

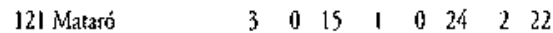

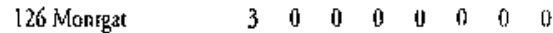

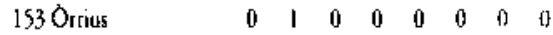

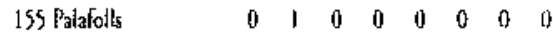

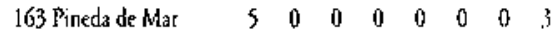

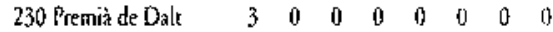

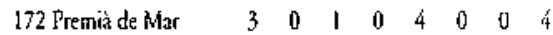

197 Sant Andreu de

Llavaneres

261 Santa Susanna

$\begin{array}{llllllll}1 & 1 & 0 & 0 & 0 & 0 & 0 & 0\end{array}$

$\begin{array}{llllllll}0 & 1 & 0 & 0 & 0 & 0 & 0 & 0\end{array}$

203 Sanı Cebriá de

Vallalta

$\begin{array}{llllllll}0 & 1 & 0 & 0 & 0 & 0 & 0 & 0\end{array}$

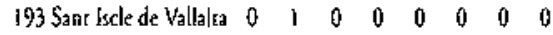

235 Sane Pol de Mar $\quad 1 \quad 1 \quad 00000000$

264 Sanr Vicenç de

Montalt

281 Teì̀

$\begin{array}{llllllllll}0 & 1 & 0 & 0 & 0 & 0 & 0 & 0\end{array}$

282 Tiana

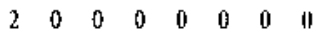

284 Tordera

214 Vilassar de Dalt

219 Vilassar de Mar $\begin{array}{llllllll}2 & 0 & 0 & 0 & 0 & 0 & 0 & 0\end{array}$

$\begin{array}{llllllll}4 & 0 & 0 & 0 & 0 & 0 & 0 & 0\end{array}$

$\begin{array}{llllllll}4 & 0 & 0 & 0 & 0 & 0 & 0 & 0\end{array}$

$\begin{array}{llllllll}7 & 0 & 0 & 0 & 0 & 0 & 0 & 0\end{array}$ 


\section{Anexo 2. Elección de variables}

El criterio más general para realizar esta elección ha sido el de tener cn cuenta su peso explicarivo o comprensivo en la totalidad del campo de aplicación y en cada ámbito, aunque no sean necesarimente independientes. Para ello se disponía de numerosos análisis previos publicados en diversos volúmenes temáticos. Para la agrupación el criterio ha consistido en asociar en un mismo cuerpo de análisis aquellas variables que se sitúan en un mismo ámbito o temática social. En total han sido 152 variablcs que intervendrán activamente en el análisis con un número de modalidades o valores $\mathrm{cn}$ total de 742. Dos de estas variables son continuas. Veamos los ámbitos y una indicación de las variables que les corresponden.

a) Se han elegido, primero, un conjunto de variables que hemos denominado identificativas o independientes. Podemos suponer que tienen un fuerte peso en la estruturación de la realidad social de la Región, no sólo por los resultados ya consuetudinarios en este tipo de estudios sino también por los análisis previos bivariados hechos en la misma Enquesta. Estas variables categóricas se refieren a:

- la posición en el mercado de trabajo del entrevistado y de su cabcza de familia,

- el sexo,

- la edad del individuo y del cabeza de familia,

- el lugar de nacimiento en función de coronas geográficas y

- el voto político emitido.

b) Otro conjunto de variables tenidas en cuenta son de catácter dependiente con relación a las primeras y que conftguran los diferentes ámbitos sociales.

En la línea del capital económico encontramos diversos ámbitos de variables quc intervienen fuertemente en el proceso de estructuración del conjunto de los datos $y$, por consiguiente, de la identidad de los grupos sociales. Están ligados a la producción, a los ingresos y a otras formas de posesiones.

1. Trabajo

- Trabajo productivo: sector de actividad, tamaño de la empresa, situación lalworal.

- Trabajo reproductivo expresado en horas dedicadas.

2. Riquezas del hogar

- Niveles de ingresos: individuales, familiares.

- Otros indicadores: inversiones, propiedades, seguros contratados, gastos cscolares.

3. Equipamiento del hogar

- Equipamiento en infraestructura.

4. Residencia

- Características de la vivienda donde se vive: superficie, antigüedad, etc.

- Posesión de segunda residencia.

En la dirección de lo que se demomina capital cultural, que comprende un conjunto de variables que tienen como teferencia los hábitos culturales y los bicnes y las capacidades educativas. Globalmente su valor estructural es considerable 
y posiblemente cada vez más importante y no absoluta o miméticamente traspasable o reducible al valor económico precedente.

\section{Educación}

- Nivel educativo reglado: del entrevistado, del hogar de origen.

- Indicadores de frecuencia de hábitos cuiturales.

Según la orientación, en parte de lo que podríamos llamar capital relacional, en parte según un conjunto de prácticas que componen el encramado de actividades cotidianas, relacionales o más generales. Su importancia es grande, en particular porque cimentan y constituyen el fluir de la vida diaria y porque son elementos de relativa inercia a su modificación y a la penetración de otros factores.

6. Relaciones personales

- Número, frecuencia y expresión de las relaciones personales.

7. Tiempo libre

- T"ipos, modalidades, uso y expresión de las actividades del ciempo libre.

8. Hábitos de consumo

- Hábitos de compra.

- Modalidad de pago.

- Movilidad debido al consumo.

9. Composición y relaciones familiares

- Tipología y organización de la familia.

10. Vacaciones

- Frecuencia, duración y modalidades de las vacaciones.

11. Origen geográfico

- Origen de los miembros del hogar.

- Lengua del entrevistado.

\section{Anexo 3. Perfil de las zonas sociales}

La zona $1+2$ : pequeños municipios fuera de Barcelona

Su especificidad proviene precisamente de estar constituida por pequeños municipios. En este sentido no tiene ninguna semejanza con el resto de las zonas. Sí que guarda una cierta similitud de otros contenidos con la media general. En ella se llegan a percibir dos tipos de población: una que parece la población de siempre, asentada, y otra de más recienre implantación, aunque es difícil, salvo en el año de llegada $y$ de construcción de la vivienda, diferenciar ambas poblaciones.

La zona 3: la media o la reducción a escala social de toda la región.

Como zona media, en parte se parece a todas y en parte se diferencia. Globalmente es una población de gran estabilidad y equilibrio en todos los ámbitos sociales aunque con tendencia a un cierto declive generacional: una cierta identidad que aumentará en el futuro. En este sentido, es una media decreciente o menguante. Es una zona afincada y enraizada y que pucde representar aproximadamente bien la imagen del ciudadano urbano medio barcelonés. 


\section{La zona 4: los ricos.}

La zona 4 representa el estatus más alto de la población de la región en todos los ámbitos. Tiene una delimitación social muy definida y está relativamente concentrada geográficamente. Es una zona no muy extensa, relativamente bien localizada. Globalmente es una población de gran coherencia entre todos los ámbitos sociales. Es palpable la idea de continuidad generacional dado que se mantienen los resortes básicos de reproducción de la zona.

Las zonas 5 y $6:$ la inmigración.

Las zonas 5 y 6 están compuestas, en su gran mayoría, por el medio inmigrante de los años cincuenta, sesenta. Ambas se convierten en el paradigma de un medio popular con características, en casi todos los indicadores, de los niveles bajo y mediobajo y a difícil remolque de su integración a la cultura catalana de recepción. Se ha de decir que la distinción entre zonas 5 y 6 , que aparecia neta en los estratos muestrales, sobre todo a partir del factor edad, no está tan clara cuando se introducen los datos de la encuesta. Se dan diferencias, pero no son suficientes como para hablar de dos zonas distintas. Si no las hemos uniffcado en una zona $5+6$ es porque aquí había suficiente consistencia muestral como para rratarlas separadamente. Ambas, globalmente, son un medio migrante proletarizado, de vida modesta, de relaciones familiares y cercanas, vinculadas al barrio en el que encuentran su autonomía y donde desarrollan su vida cotidiana. La zona 5 es menos cerrada al medio que la 6 . Viven en la precaridad que produce la inseguridad de los tiempos que corren, ta zona 6 quizás está más inmersa aún en dicha incertidumbre.

Zona 7: los mayores de bajos estatus

La Zzona 7 contiene también una población de base inmigrante antigua, aunque el paisaje urbano y la caracterización social no sean similares a las precedentes. Es una población inmigrante de hace muchos años, más altá de las grandes oleadas migratorias: son de ahi, son de siempre, aunque muchos sean de fuera, y son mayores. Es una zona popular que se encuentra en los límites de la marginación social. Afincados a un barrio que es, en muchos de los casos, lo único que les queda en la soledad de sus hogares unipersonales: viudos, mayores, sin hijos y con los índices más bajos cn todos los ámbitos sociales. Es evidente, como sabemos, que no toda la zona es así, pero es el perfil más prominente. La vivienda y el entorno son, pues, importantes para esta población siendo parte de su especificidad.

La zona 8: los inicios a la vida profesional de un medio bien situado

La zona 8 representa globalmente un alto estatus y es una de las más dinámicas desde el punto de vista de movilidad social, geográfica y de edad. Es una población emergente, de gran ocupación, profesionales preparados, sobre todo técnicos, parcialmente proveniente de la segunda generación y de movilidad ascendente, tanto $\mathrm{cn}$ estatus como generacional y en nivel educativo. Tiene signos evidentes de modernidad en los aspectos profesionales, en el estilo de vida, en el tipo de familia, en el trabajo de la mujer, en el tipo de relaciones asociativas que mantiene, en la disponibilidad y ocupación del tiempo libre, etc. La familia es reducida. Sin olvidar que rambién se dan en su interior otras capas de la población. 
Anexo 4.

Ilustración de

la distribución

de las zonas sociales

en el distrito 6:

Gràcia.

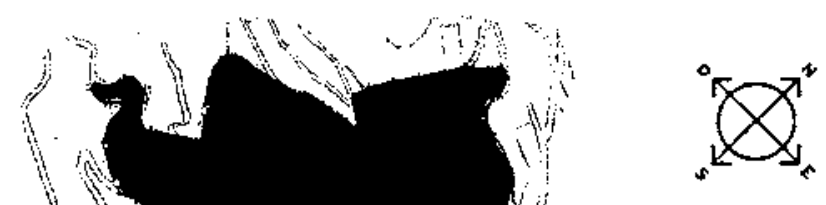

$\therefore$ Zona dels nuclis urbans grans, corresponde a la zona 3.

Zona de població benestant,

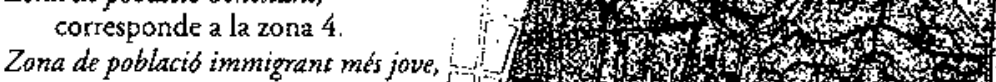
corresponde a la zona $S$.

Zona de població immigrant més granis. corresponde a la zona 6.

Zona de població vella, corresponde a la zona 7 .

Zona de nova clase mitjana jove, corresponde a la zona 8 .

\section{a la zona 8.}

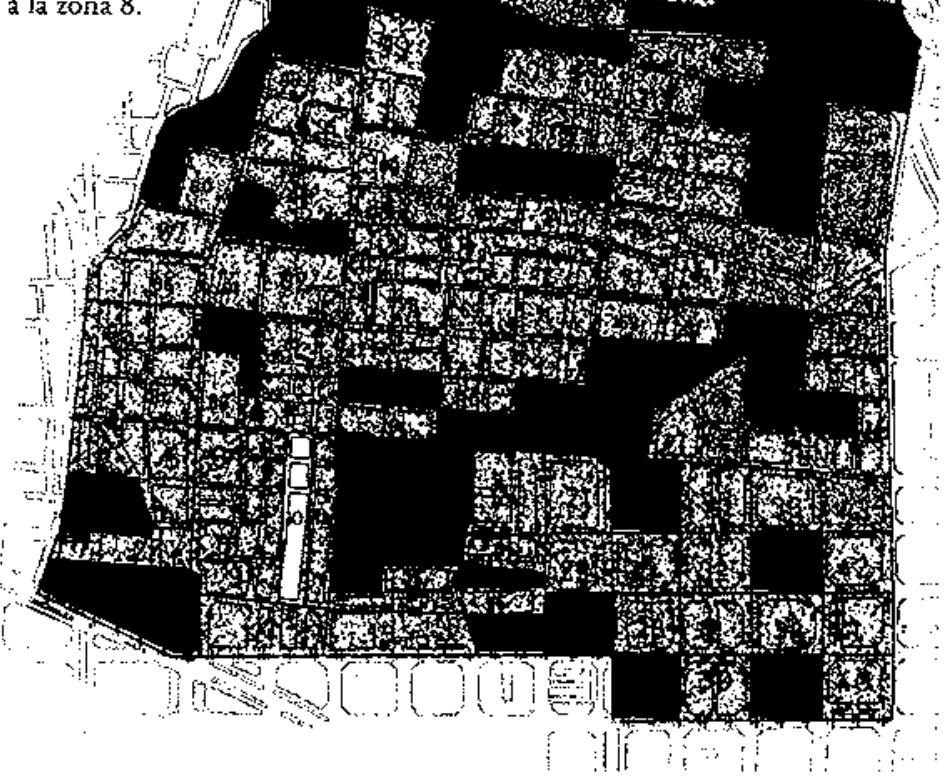




\section{Referencias}

LOZARES, Carlos; LOPEZ, Pedro (1991a). "El muestreo estratiftcado por análisis multivariado". En Latiesa, M. El pluralismo metodológico en la investigación social: Ensayos tipicos. Granada: Universidad de Granada, p. 107-160.

- (1991b). "El análisis de componentes principales: aplicación al análisis de datos secundarios". En Papers 37, 1991: 31-64.

LozARES, Carlos; DomínGUez, Màrius (1993). "Territori i realitat social: les zones socio-demogràfiques de la Regió Metropolitana de Barcelona". Vol. 9 de Enquesta de la Regib Metropolitana de Barcelona 1990: Condicions de vida i hàbits de la població. Barcelona: Institut d'Estudis Merropolirans.

Subirats, Marina; SÁNChez, Crisrina; Dominguez, Màrius (1992). "Grups i classes socials a la Regió Metropolitana de Barcelonan. Vol. 5 de Enquesta de La Regió Metropolitana de Barcelona 1990: Condicions de vida i hàbits de La població. Barcelona: Insitur d'Esrudis Merropolitans. 